\title{
Markov Chain based Emissions Models: a Precursor for Green Control
}

\author{
E. Crisostomi ${ }^{1}$, S. Kirkland ${ }^{2}$, A. Schlote ${ }^{2}$, and R. Shorten ${ }^{2}$ \\ ${ }^{1}$ Department of Energy and Systems Engineering, University of Pisa, Italy, \\ email: emanuele.crisostomi@gmail.com \\ ${ }^{2}$ Hamilton Institute, National University of Ireland - Maynooth, Co. Kildare, Ireland, \\ email: \{stephen.kirkland, arieh.schlote, robert.shorten\}@nuim.ie
}

\begin{abstract}
In this chapter we propose a new method of modelling urban pollutants arising from transportation networks. The efficacy of the proposed approach is demonstrated by means of a number of examples. Our models give rise to a number of surprising observations that are relevant for the regulation of pollution in urban networks: Different actions are required for the control of different pollutants and low speed limits do not necessarily lead to low pollution.
\end{abstract}

\section{Introduction}

The issue of Green Control is likely to become a major research topic over the next decade. Roughly speaking, this term refers to the development of control strategies for deployment in large scale networks that can be used to regulate, share and optimize the use of quantities related to "green systems". Generally speaking, these "green variables" might include atmospheric pollutants, carbon emissions, energy usage and reuse. One area, in which activity in "green control" is already underway, is in the regulation of pollution and emissions in urban transportation networks. In the automotive industry, public awareness of the link between greenhouse gasses and road transportation is great. While $\mathrm{CO}_{2}$ emissions are ultimately harmful to humans through ozone depletion, road transportation is also very harmful to humans in a direct manner. According to a UK study (FoE 1999), road transportation's percentage contribution to air pollution in 1999 was: $80 \%$ in the case of $\mathrm{CO} ; 75 \%$ in the case of benzene; $50 \%$ in the case of NOx; $40 \%$ for hydro carbons (producing ozone); and $25 \%$ of particulates, all of which are extremely harmful to humans. See (Levy et al. 2010) for details of established sideeffects of these pollutants. It is also important to note recent advances in health 
science where the link between heart attack triggers and urban pollution is hypothesized (Nawrot et al. 2011).

Traditionally, researchers have advocated two approaches to reducing air pollution: the production of greener cars; and an overall reduction in road transportation. These involve not only introducing laws that produce ever more stringent regulations on engine manufacturers, but also some cities are trying to regulate pollution in certain areas through control policies. The city of Berlin, Germany, is one such example, where strict open-loop control policies based on clean engines are implemented.

An alternative approach is to investigate advances in ICT for the automobile sector to control and regulate emissions and pollution in our cities. This latter approach builds on three policy directions coming from regulatory bodies (such as the EU and the US government) aimed at (1) reducing greenhouse gasses, (2) reducing pollution peaks in our cities, and (3) developing instrumentation, cooperative control strategies, and modelling techniques to enable the development of proactive traffic management systems, i.e. systems that predict traffic flow and take pre-emptive measures to avoid incidents (traffic build up, pollution peaks etc). It is also consistent with strategic developments in the automotive and networking industry where the control and regulation of large scale systems is seen as a priority objective in company roadmaps. Initiatives in this direction include the IBM smarter city initiative and the CISCO smart and connected communities programme, as well as the many initiatives underpinning vehicle to vehicle communications and vehicle to infrastructure communications.

Our objective in this work is more modest. While our ultimate objective is control and optimization, we are motivated by the fact that underpinning every good control strategy is an appropriate model. Our starting point is a recently proposed Markovian approach to modelling road network dynamics (Crisostomi et al. 2011). As discussed in (Crisostomi et al. 2011), a Markovian framework is particularly appealing since it makes important information regarding the road network available in a convenient form to the road network designer (congestion, average travel times, sensitive links in the road network). It is also useful for control and optimization applications. Our objective in this work is to demonstrate how this model can be extended to build emissions models, and to use this model to inform what might and might not be possible in a control setting. As we shall see, inference from the models will give rise to counter intuitive facts that must be taken into account when developing policy to reduce air pollution and emissions.

\section{Macroscopic models and Markov Chains}

Our approach, to be described later, is based on using Markov chains to model a large scale and highly complicated dynamic system. At a first glance this approach may seem strange and overly optimistic. However, using Markov chains to model 
highly complex dynamic systems has been successful in the Mathematics and Engineering communities. To give the reader a flavour of this prior work we now describe briefly two applications.

As a first example, we consider a problem from astrophysics; namely, of a planar circular restricted three-body problem. This is a special case of the three body problem which has inspired a lot of advances in dynamic systems. Here two of the three bodies are assumed to have significantly more mass than the third and of these two bodies - the lighter one - is orbiting the heavier one on a circular trajectory. The third body is free to move around the state space and it is assumed to be without mass. The movement of all three bodies is assumed to be restricted to a plane. We can imagine this as a planet that has a moon on a circular orbit and the third body is an asteroid whose motion is restricted to the plane in that the moons orbit lies. In (Koon et al. 2000,2002) the planar circular restricted three-body problem is analysed by partitioning the state space into three regions: an interior and an exterior region with respect to the moon, and a region close to the moon, where the asteroids motion is governed by the moon's gravitational force. The authors show that for any sequence of these regions they can find a possible orbit of the asteroid that will move through the regions in the given sequence. This is done by identifying a number of important system equilibria and corresponding invariant manifolds. The existence of homoclinic and heteroclinic orbits is discussed. Chains of these orbits can be constructed such that solutions close to these chains visit the regions in the given sequence. This requires advanced methods from dynamic systems theory. In (Dellnitz et al. 2005) it is shown how the results can be refined using a much simpler approach using Markov chains.

Another example is what is known as the transfer operator approach to modelling dynamic systems. Given a Markov process on some state space and some probability distribution on it, the Frobenius-Perron operator determines the evolution of the probability measure in time. Invariant measures of the system can be identified with eigenfunctions of the Frobenius-Perron operator to the eigenvalue 1. In (Dellnitz and Junge 1999) the spectrum of the Frobenius-Perron operator is numerically approximated by analysing the spectrum of discrete approximations to the Frobenius-Perron operator. This is successfully applied to model the dynamics of complex bio molecules, see (Huisinga 2001) and (Schütte and Huisinga 2003). The idea here is to cut the state space into a finite number of regions. The importance of these regions for the system dynamics can be assessed by creating a Markov chain with the regions as states and to determine the transition probabilities one uses a transfer operator. This operator does not depend on the choice of the partition. Moreover analysing the operator's spectrum and the eigenspaces for a certain range of eigenvalues yields information about almost invariant sets and allows one to find a clever way of partitioning the state space. Almost invariant sets are subsets of the state space where solutions stay for a long time and can often be identified with important properties of the modelled system. Insight can be 
gained by using a Markov chain that models these almost invariant sets and the transition rates between them.

A very good and compact introduction to modelling complex dynamic systems as Markov chains can be found in (Froyland 2001). A lot of the information about ergodic systems is contained in the distribution of a typical long trajectory, also called the system's natural measure. It is shown how this natural measure can be approximated using Markov Chains.

In our specific case, traffic in an urban network is a highly complicated dynamical system that can, more often than not, be accurately modelled only by using equally complicated models. However, the network planner is usually interested in aggregation effects (pollution, congestion, travel times etc.). We believe that we have found a way to use a very simple Markov chain model, that allows us to compute important properties of such a network with high accuracy and very high speed. This was first described in (Crisostomi et al. 2011), and is further developed here to model pollution dynamics.

\section{A primer on Markov chains}

The objective of this section is to present the mathematical tools that will be used in the rest of the chapter. The preliminary definitions are standard and can be found in classic references like (Meyn and Tweedie 2009) or, in a short summary, given in (Langville and Meyer 2006, Chapter 15). Here we give only the most basic concepts that are needed for our discussion.

Recall that a Markov chain is a stochastic process characterised by the equation

$$
p\left(x_{k+1}=S_{i_{k+1}} \mid x_{k}=S_{i_{k}}, \ldots, x_{0}=S_{i_{0}}\right)=p\left(x_{k+1}=S_{i_{k+1}} \mid x_{k}=S_{i_{k}}\right)
$$

where $p(E \mid F)$ denotes the conditional probability that event $E$ occurs given that event $F$ occurs. Equation (1) states that the probability that the random variable $x$ is in state $S_{i_{k+1}}$ at time step $k+1$ only depends on the state of $x$ at time step $k$ and not on preceding values. Throughout the chapter only discrete-time, finite-state, homogeneous Markov chains will be considered. We present no theoretical justification for our model, other than to state that Markov chains have a long history of providing compact representations of large scale systems described by very complicated sets of dynamical equations, as already illustrated in the previous section.

The Markov chain is completely described by the $n \times n$ transition probability matrix $\mathbf{P}$ whose entries $\mathbf{P}_{\mathrm{ij}}$ denote the probability of passing from state $S_{i}$ to state 
$S_{j}$, and $n$ is the number of states. The matrix $\mathbf{P}$ is a row-stochastic non-negative matrix, as the elements of each row are probabilities and they sum up to 1 . Within Markov chain theory, there is a close relationship between the transition matrix $\mathbf{P}$ and its corresponding graph. A graph is represented by a set of nodes that are connected through edges. Therefore, the graph associated with the matrix $\mathbf{P}$ is a directed graph, whose nodes are represented by the states $S_{i}, i=1, \ldots, n$ and there is a directed edge leading from $S_{i}$ to $S_{j}$ if and only if $\mathbf{P}_{\mathrm{ij}} \neq 0$.

A graph is strongly connected if and only if for each pair of nodes there is a sequence of directed edges leading from the first node to the second one. The matrix $\mathbf{P}$ is irreducible if and only if its directed graph is strongly connected. Let us now state the well-known Perron-Frobenius theorem (Langville and Meyer 2006), which summarises important properties of irreducible transition matrices:

- The spectral radius of $\mathbf{P}$ is 1

- 1 also belongs to the spectrum of $\mathbf{P}$, and it is called the Perron root

- The Perron root has an algebraic multiplicity of 1

- The left-hand Perron eigenvector $\pi$ is the unique vector defined by $\boldsymbol{\pi}^{\mathbf{T}} \mathbf{P}=\boldsymbol{\pi}^{\mathbf{T}}$, such that $\boldsymbol{\pi}>\mathbf{0},\|\boldsymbol{\pi}\|_{\mathbf{1}}=\mathbf{1}$. Except for positive multiples of $\boldsymbol{\pi}$ there are no other non-negative left eigenvectors for $\mathbf{P}$.

In the last statement, by saying that $\boldsymbol{\pi}>\mathbf{0}$, it is meant that all entries of vector $\pi$ are strictly positive. One of the main properties of irreducible Markov chains is that the $i^{\text {th }}$ component $\boldsymbol{\pi}_{\mathbf{i}}$ of the vector $\boldsymbol{\pi}$ represents the long-run fraction of time that the chain will be in state $S_{i}$. The row vector $\pi^{\mathrm{T}}$ is also called the stationary distribution vector of the Markov chain.

\subsection{Mean first passage times and the Kemeny constant}

A transition matrix $\mathbf{P}$ with 1 as a simple eigenvalue gives rise to a singular matrix I-P (where the identity matrix I has appropriate dimensions) which is known to have a group inverse $(\mathbf{I}-\mathbf{P})^{\#}$. The group inverse is the unique matrix such that $(\mathbf{I}-\mathbf{P})(\mathbf{I}-\mathbf{P})^{\#}=(\mathbf{I}-\mathbf{P})^{\#}(\mathbf{I}-\mathbf{P}), \quad(\mathbf{I}-\mathbf{P})(\mathbf{I}-\mathbf{P})^{\#}(\mathbf{I}-\mathbf{P})=(\mathbf{I}-\mathbf{P}) \quad$ and $(\mathbf{I}-\mathbf{P})^{\#}(\mathbf{I}-\mathbf{P})(\mathbf{I}-\mathbf{P})^{\#}=(\mathbf{I}-\mathbf{P})^{\#}$. More properties of group inverses and their applications to Markov chains can be found in (Meyer 1975). The group inverse $(\mathbf{I}-\mathbf{P})^{\#}$ contains important information on the Markov chain and it will be often used in this chapter. For this reason, it is convenient to denote this matrix as $Q^{\#}$. 
The mean first passage time $m_{i j}$ from the state $S_{i}$ to $S_{j}$ is the expected number of steps to arrive at destination $S_{j}$ when the origin is $S_{i}$. If we denote $q_{i j}^{\#}$ as the $i j$ entry of the matrix $Q^{\#}$, then the mean first passage times can be computed easily according to the equation below (see Cho and Meyer 2001)

$$
m_{i j}=\frac{q_{j j}^{\#}-q_{i j}^{\#}}{\pi_{j}}, \quad i \neq j
$$

where it is intended that $m_{i i}=0, i=1, \ldots, n$. The Kemeny constant is defined as

$$
K=\sum_{j=1}^{n} m_{i j} \pi_{j}
$$

where the right hand side is (surprisingly) independent of the choice of $i$ (Kemeny and Snell 1960). Therefore the Kemeny constant $K$ is an intrinsic measure of a Markov chain, and if the transition matrix $\mathbf{P}$ has eigenvalues $\lambda_{1}=1, \lambda_{2}, \ldots, \lambda_{n}$ then another way of computing $K$ is (see (Levene and Loizou 2002))

$$
K=\sum_{j=2}^{n} \frac{1}{1-\lambda_{j}} .
$$

Equation (4) emphasizes the fact that $\mathrm{K}$ is only related to the particular matrix $\mathbf{P}$ and that it increases if one or more eigenvalues of $\mathbf{P}$ is real and close to 1 .

\section{From a road network model to a pollution model}

The use of Markov chains to model road network dynamics has been described in detail in (Crisostomi et al. 2011). The resulting networks are fully characterized by a transition matrix $\mathbf{P}$, which has the following form: 


$$
\mathbf{P}=\left[\begin{array}{cccc}
P_{S_{1} \rightarrow S_{1}} & P_{S_{1} \rightarrow S_{2}} & \ldots & P_{S_{1} \rightarrow S_{n}} \\
P_{S_{2} \rightarrow S_{1}} & P_{S_{2} \rightarrow S_{2}} & \ldots & P_{S_{2} \rightarrow S_{n}} \\
\vdots & \vdots & \ddots & \vdots \\
P_{S_{n} \rightarrow S_{1}} & P_{S_{n} \rightarrow S_{2}} & \ldots & P_{S_{n} \rightarrow S_{n}}
\end{array}\right] .
$$

The matrix $\mathbf{P}$ is a square matrix whose size is given by the number of road segments. The off-diagonal elements $P_{S_{i} \rightarrow S_{j}}$ are related to the probability that one passes directly from the road segment $S_{i}$ to the road segment $S_{j}$. They are zero if the two road segments are not directly connected (i.e. at the end of road $S_{i}$ it is impossible to take directly road $S_{j}$ ). The diagonal terms are proportional to travel times; namely, they are close to 1 if it takes a long time to cover the corresponding road while they are closer to 0 if travel times are short. The diagonal terms take into account several factors that affect travel times; such as speed limits, road surface conditions, presence of priority rules or traffic lights, weather conditions, heavy traffic, etc. The diagonal elements can be computed from average travel times through the following equation

$$
P_{S_{j} \rightarrow S_{j}}=\frac{t t_{j}-1}{t t_{j}}, \quad j=1, \ldots, n,
$$

where $t t_{j}$ indicates the average travel time along the $j^{\text {th }}$ road.

The main idea of this work is to use the same framework to model pollutants, This can be achieved by replacing time in the original chain described above, by a unit of pollutant (e.g. benzene, NOx, etc...). In this framework, a car is moving in the same road network, and changes (or remains in the same) state anytime a unit of pollutant is released, according to the entries of a second transition matrix $\mathbf{P}_{\mathbf{P}}$, where the subscript $\mathrm{P}$ stands for pollution. The quantity of emissions released along a particular road $S_{j}$ does not only depend on travel times, but also on other quantities like the length of the road, speed profile along the road (i.e. number of times the car accelerates or decelerates) and average types of car in the particular road (fuel type, engine capacity, etc). As a consequence, the main difference between the chain described in (Crisostomi et al. 2011) and the chain here are the diagonal entries of matrix $\mathbf{P}_{\mathbf{P}}$.

It is important to note that we are proposing a new paradigm for pollution modelling that captures large scale (macroscopic) effects. For the purpose of constructing a model, and its evaluation, a microscopic model to describe microscopic effects is required. There are of course several models to evaluate pollution at 
microscopic level, at very different levels of accuracy and complexity (Jaaskelainen and Boethius 2009). A first basic model is the so-called aggregated emission factor model, where a single emission factor is used to represent a particular type of vehicle and a general type of driving, with a usual distinction between urban roads, rural roads and motorways. This model is rather rudimentary and is not realistic for small scale networks, as it omits several phenomena, such as congestion, which are known to significantly affect emissions. In this work, we shall make use of more refined average-speed models to build our macroscopic Markov model, where emission factors are calculated as a function of average speed (Barlow et al. 2001) and (Boulter et al. 2009). The average-speed approach is described in the UK Design Manual for Roads and Bridges (DMRB) (Highways Agency et al. 2007) and the European Environment Agency's COPERT model (Gkatzoflias et al. 2007).

Although the average-speed model has been extensively used for many applications, it suffers from a drawback that very different vehicle operational behaviours (in terms of accelerations, decelerations, maximum speed, gear-change pattern), and therefore different emission levels, can be characterized by the same average speed. A more realistic model is for instance the comprehensive modal emissions model (CMEM) described in reference (Barth et al. 2000). According to this model, second-by-second exhaust emissions and fuel consumption are predicted, for a wide range of vehicle categories and ages. For the sake of simplicity, in this work the average-speed model is employed. However, the same proposed methodology can be applied in combination to any other (more accurate) vehicle emissions model.

\subsection{Construction of the Markov chain transition matrix}

The emissions model is completely determined by a transition matrix $\mathbf{P}_{\mathbf{P}}$, whose diagonal terms indicate how many units of pollution a fleet of vehicles releases along a road segment, and by off-diagonal terms that indicate the next road segment chosen by a unit of pollution. These entries are computed as follows.

Diagonal terms: According to average-speed models, the emission factor $f(t, p)$ is computed as

$$
f(t, p)=\frac{k \cdot\left(a+b v+c v^{2}+d v^{3}+e v^{4}+f v^{5}+g v^{6}\right)}{v},
$$

where $t$ denotes the type of vehicle (and depends on fuel, emission standard, category of vehicle, engine power), $p$ denotes the particular type of pollution of inter- 
est (e.g. $\mathrm{CO}, \mathrm{CO}_{2}, \mathrm{NOx}$, Benzene), $v$ denotes the average speed of the vehicle, and the parameters $a, b, c, d, e, f, g$ and $k$ depend on the type of vehicle. For the purpose of this work, the values of the parameters are taken from Appendix D, in reference (Boulter et al. 2009). In Equation (7) it is assumed that speeds are measured in $\mathrm{km} / \mathrm{h}$ and emission factors in $\mathrm{g} / \mathrm{km}$. Therefore, by assuming that the average speed of a fleet of vehicles along a road segment is $v$, and the length of the road segment is $l$, then the diagonal terms are given by

$$
P_{S_{j} \rightarrow S_{j}}=\frac{f(t, p)_{j} \cdot l_{j}-1}{f(t, p)_{j} \cdot l_{j}}, \quad j=1, \ldots, n
$$

by analogy with Equation (6). For simplicity we first normalize all terms $f(t, p)_{j} l_{j}$ so that the minimum one has unit value. In Equation (8) note that the emission factor depends on the road segment as its primary dependence is on the average speed along the particular road segment.

Off-diagonal terms: The Markov chain models an average car that travels in the urban network while releasing units of pollution. Therefore, it is necessary to measure the average junction turning probabilities to build the off-diagonal terms.

\subsection{The role of Information Technology in the model}

The proposed emission model requires the following items to be useful:

- The categories and types of vehicles present in each road of the network, as they are required to use the appropriate parameters in Equation (7).

- The average travel times of the vehicles along each road of the network; once travel times are known, by using the knowledge of the length of the road segments, it is possible to compute the average speeds to be used in Equation (7).

- The junction turning probabilities, which are required to build the offdiagonal terms of matrix $\mathbf{P}_{\mathbf{P}}$.

- Centralized (or decentralized) number crunching ability to calculate properties of the large scale matrix.

There are two possible ways of obtaining the information required for the Markov chain: 
1. All cars are instrumented to behave as mobile sensors and store the data relative to their travelling history. At regular times, they communicate their data to a central database that collects all the important information.

2. Urban networks are equipped with loop detectors that are positioned at each junction, and they measure the required information to build the Markov transition matrix.

We emphasise here that most of the required information can be easily collected (when it is not already available) by the cars themselves, and all that is required is to collect and integrate such information to construct the model. Current vehicles are memory-less. However, given the widespread penetration of vehicle positioning systems (GPS), it is easy to imagine geospatial tagging of vehicle route information, and storing this information locally in vehicle memory. It is also possible to obtain information of the type required from special classes of vehicles (buses, taxis etc.) - though this probably contains less useful information. Note such instrumented fleets already exist in cities such as Stockholm (Biem et al. 2010). Furthermore, recent advances in the development of Vehicular Adhoc NETworks (VANET) (Hartenstein and Laberteaux, 2010) are expected to further facilitate the proposed emissions model, as it is very easy to collect the information required to build the transition matrix.

Remark: CMEM (see Section 4) is one of the most accurate microscopic emission models, as it takes into account the instantaneous speed and the engine operational mode. The proposed Markovian approach can easily integrate such an emissions model. However, a high price must be paid in terms of communication between vehicles and infrastructure, as the whole speed profile of individual vehicles is required to be transmitted.

\section{Green interpretation of the Markov chain quantities}

Once the transition matrix has been constructed, it is very easy to infer several quantities of potential interest to the designer of a road network. These include the Perron eigenvector, the mean first passage time matrix and the Kemeny constant. The proposed model has the property that it is driven by a unit of pollution rather than by a unit of time as in conventional Markov chains. For ease of exposition in the present discussion we use a generic unit of pollutant, while in the simulation examples we will compute $\mathrm{CO}, \mathrm{CO}_{2}$, $\mathrm{NOx}$ and Benzene. We remind the reader that the difference between the models is in the choice of the parameters in Equation (7). The next table summarises the interpretation of the Markov chain quantities in the emission framework. 
Table 1 Interpretation of Markov chain quantities in the emission framework

\begin{tabular}{ll}
\hline Markov chain quantity & Green interpretation \\
\hline Left-hand Perron Eigenvector & $\begin{array}{l}\text { This vector has as many entries as the number of road } \\
\text { segments. Each entry represents the long run fraction } \\
\text { of emissions that a fleet of vehicles will emit along the } \\
\text { corresponding road segment. It can be used as an indi- } \\
\text { cator of pollution peaks. }\end{array}$ \\
Mean first passage emissions & $\begin{array}{l}\text { This is a square matrix with as many rows as the num- } \\
\text { ber of the road segments. The entry } \text { ij represents the } \\
\text { expected quantity of emissions that a vehicle releases } \\
\text { to go from } i \text { to } j \text {. The average is with respect to all pos- } \\
\text { sible paths from } i \text { to } j .\end{array}$ \\
Kemeny constant & $\begin{array}{l}\text { This number is the average number of emissions re- } \\
\text { leased in a random route. It is an indicator of pollution } \\
\text { in the entire network. }\end{array}$
\end{tabular}

There are other quantities that can be computed within the proposed framework:

Density of emissions along each road (g/km): They can be easily computed from Equation (7): it is only required to know the average fleet of vehicles and the average speed along the road.

Emissions along each road (g): They can be easily computed by multiplying the density of emissions along a road by the length of the road.

Total Emissions (g): It is sufficient to sum the emissions along each road for all the roads inside the area of interest (e.g. the urban network).

\section{Examples}

In this section the proposed approach is described in detail through several simulations. The information required to build the Markov transition matrix is recovered from simulating traffic within an urban network using the well-known mobility simulator SUMO (Simulation of Urban MObility) (Krajzewicz et al. 2006). SUMO is an open source, highly portable microscopic traffic simulation package that was developed at the Institute of Transportation Systems at the German Aerospace Center, and is licensed under the GPL. Simulations are required to extract junction turning probabilities and average travel times along the road segments composing the urban network. Three steps must be performed to achieve this goal: 
1. Creation of the urban network: this includes the topology of the network, the use of traffic lights or priority rules, fixing speed limits, choosing the number of lanes, etc.

2. A random pair of origin/destination roads is assigned to each car. The car will travel toward its destination according to the minimum length path.

3. Traffic statistics, namely junction turning probabilities and average travel times, are collected from the urban network and used as the data from which we build the Markovian emissions model.

We now give two examples of road networks to demonstrate the usefulness of our approach.

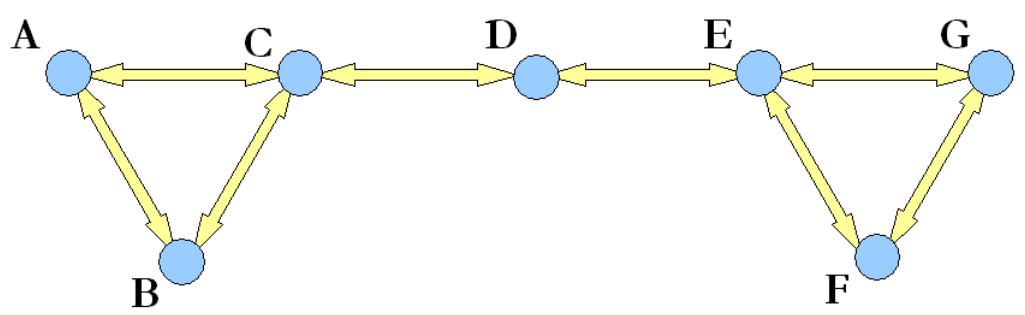

Fig. 1 Graphical representation of a simple urban network. Nodes and edges correspond to junctions and road segments respectively. Bidirectional arrows show that both travelling directions are allowed.

Consider the road network depicted in Figure 1. Here the road network is represented as a graph with nodes corresponding to road intersections and edges corresponding to streets between the intersections, we will call this representation the primal graph (Porta et al. 2006). To use the analytical tools as described in Section 3, we need a different representation of the network called the dual graph. In the dual graph the nodes correspond to streets and there is an edge between two nodes if it is possible to continue from the first road to the second road. The dual graph thus contains information that is not accessible from the primal graph. As an example, a dual representation of the primal graph shown in Figure 1 is illustrated in Figure 2. Unless stated otherwise all road segments have a length of 500 meters. 


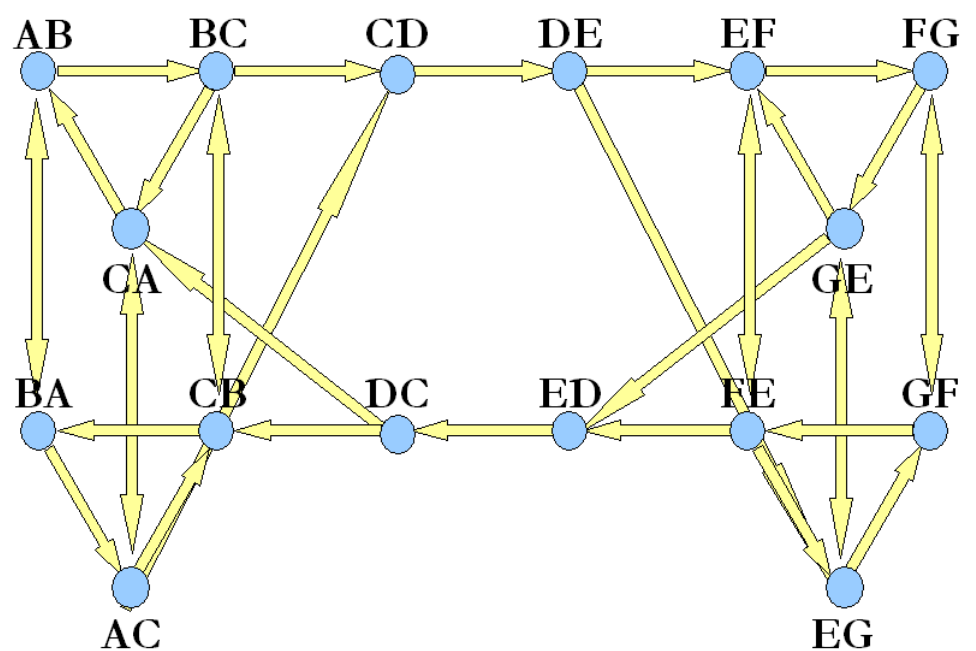

Fig. 2 Dual representation of the primal network shown in Figure 1. For instance, the road segment $\mathrm{AB}$ is the (directed) road that goes from junction $\mathrm{A}$ to $\mathrm{B}$ in the primal network.

SUMO is now used to create traffic inside the urban network described in Figures 1 and 2, in two different operating conditions. In the first case the number of cars in the road network is small, and cars are allowed to travel freely at maximum allowed speed; in the second case heavy congestion is artificially simulated by increasing the number of cars, so that actual speeds are slower than the maximum allowed by speed limits. The computation of emissions is performed according to Equation (7), using the data corresponding to Euro 4, Engine Capacity < $1400 \mathrm{cc}$ petrol cars and minibuses with weight below 2.5 tonnes (Code R005/U005 from Boulter et al. 2009).

Figure 3 shows that if the emissions are computed using speed-independent emission factors, then they are not affected by different levels of congestion. This is clearly absurd. In fact, the stationary distribution of emissions is the same, both in the figure on the left (no congestion), and in the one on the right (congestion). On the other hand, the stationary distribution of cars along the roads (represented with the solid line) changes completely in the case of congestion. 

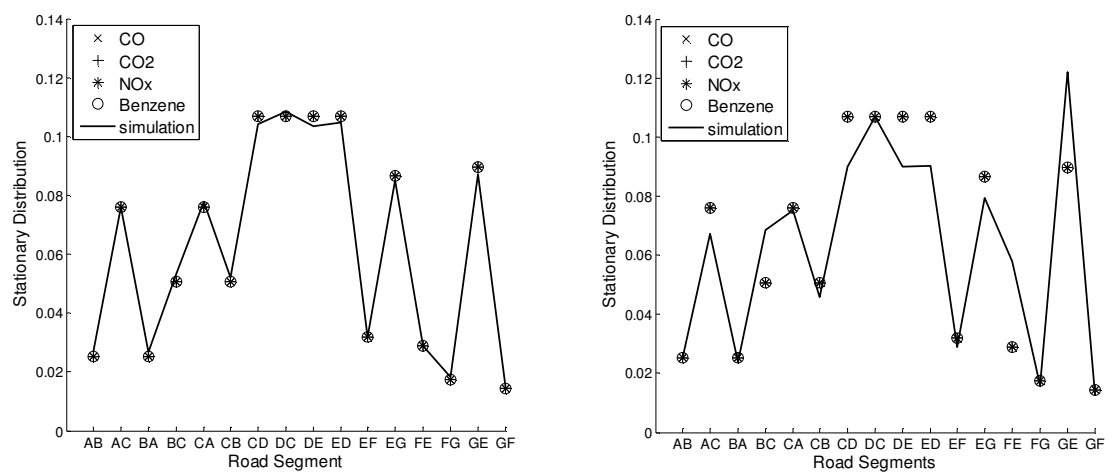

Fig. 3 Stationary distribution of cars (solid line) and all pollutants. Pollutant factors are constant with speed. No congestion on the left, congestion on the right. All emission factors coincide because of density normalisation (their sum is 1).
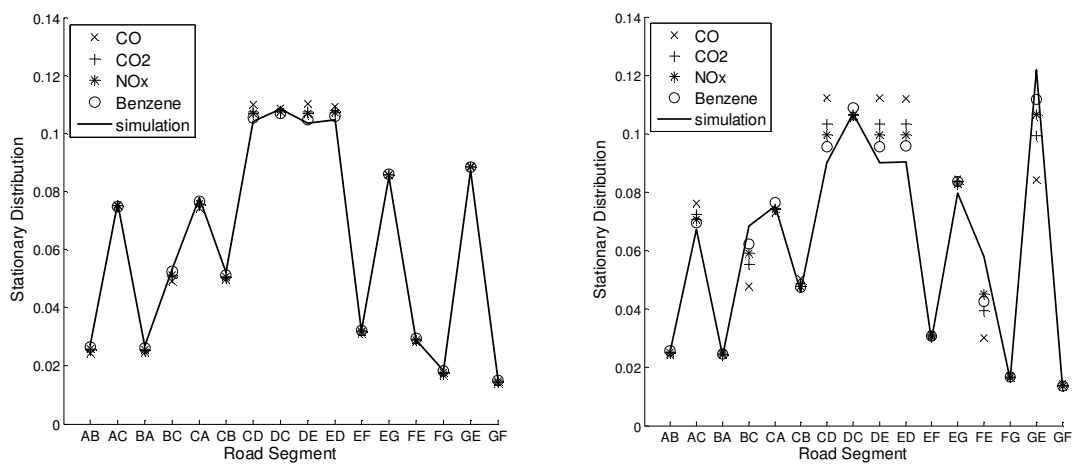

Fig. 4 Stationary distribution from simulation and model for time and all pollutants. Pollutant factors are dependent on speed. In this example, the pollutants density follows the car density both in the non-congested scenario (left) and in the congested one (right).

In Figure 4 the pollution factors are assumed to depend on velocity, and therefore the stationary distribution of emissions changes from the non-congested (left) to the congested scenario (right), and it remains consistent with the distribution of cars. In both Figures 3 and 4, and in both scenarios, the junction turning probabilities are the same. Clearly, it is not sensible that the distribution of pollutants does not change with traffic load. Therefore, this simple simulation suggests that speeddependent emission factors should be used to obtain realistic results. In Figure 4, we can also see that the manner in which pollutants are influenced by the traffic 
volume is not homogeneous with respect to congestion (i.e. different pollutants have different density).

A new simulation is now performed to establish optimal speed limits. Optimality is with respect to minimum emissions. The results are shown in Table 2. Within each simulation we varied the speed limit uniformly over all streets from 20 to $120 \mathrm{~km} / \mathrm{h}$ and calculated the corresponding Kemeny constant. As previously described, the Kemeny constant can be interpreted as an efficiency indicator, in terms of emissions, of the overall road network.

Table 2 Kemeny Constants for Different Global Speed Limits.

\begin{tabular}{l|lllll}
\hline Speed $[\mathrm{km} / \mathrm{h}]$ & Time $[\mathrm{sec}]$ & $\mathrm{CO}[\mathrm{g}]$ & $\mathrm{CO}_{2}[\mathrm{~kg}]$ & NOx $[\mathrm{g}]$ & Benzene $[\mathrm{g}]$ \\
\hline 20 & 1304 & 2674 & 1241 & 395.9 & 2.89 \\
40 & 631 & $\mathbf{2 3 5 0}$ & 875 & 243.4 & 2.05 \\
60 & 437 & 2830 & $\mathbf{8 1 2}$ & 207.8 & 1.44 \\
80 & 352 & 3758 & 815 & 197.2 & 1.03 \\
100 & 311 & 4888 & 833 & $\mathbf{1 9 4 . 6}$ & $\mathbf{0 . 9 1}$ \\
120 & $\mathbf{2 9 1}$ & 6065 & 853 & 194.9 & 1.07 \\
\hline
\end{tabular}

Two lessons can be observed from Table 2.

(i) Too low, and too high a speed limit, both lead to high levels of pollution (although travel times are reduced with high speed limits, as expected)

(ii) Different pollutants have different corresponding optimal speed limits.

To better interpret the results shown in Table 2, Figure 5 shows how the Benzene (left) and CO (right) emission factors depend on the average speed. The speeds corresponding to minimum Benzene emissions in Figure 5 and Table 2 do not coincide. This is caused by the fact that the average speed of a car is not equal to the speed limit (e.g. due to acceleration and breaking entering and leaving the road). In this example, high speed limits helped the car to accomplish an average speed closer to the optimal value for minimum emissions. 

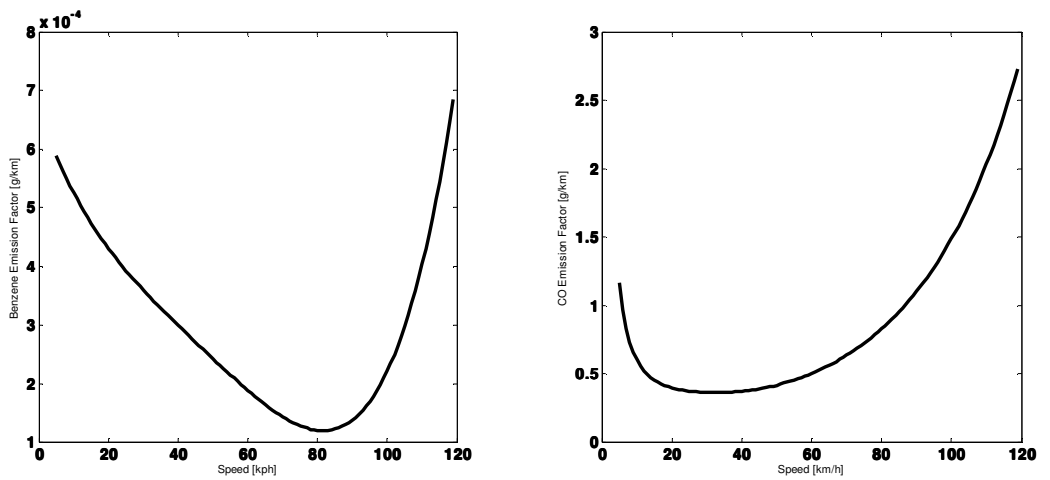

Fig. 5 Dependence of emission factor on average speed for Benzene and CO (based on data from Boulter et al. 2009).

The above experiment was repeated with one minor change. Now we vary the speed limit at certain important points in the network, and observe the corresponding global change in pollution. Specifically, we vary the speed limit of the roads CD, DC, DE and ED from Figures 1 and 2. In spirit, this corresponds in considering the urban network of Figures 1 and 2 as composed of two clusters of roads, one on the left and one on the right, connected through some "bridging" roads. Here we changed the length of the bridging roads from $500 \mathrm{~m}$ to $5 \mathrm{~km}$ - to reduce the gap between the actual average speed from the actual speed limit. Then, we vary the speed limit on the bridge from 20 to $120 \mathrm{~km} / \mathrm{h}$ while keeping the other speed limits constant.

Table 3 Kemeny Constants for Different Speed Limits on the Bridge.

\begin{tabular}{l|lllll}
\hline Speed $[\mathrm{km} / \mathrm{h}]$ & Time $[\mathrm{sec}]$ & $\mathrm{CO}[\mathrm{g}]$ & $\mathrm{CO} 2[\mathrm{~kg}]$ & NOx $[\mathrm{g}]$ & Benzene $[\mathrm{g}]$ \\
\hline 20 & 1929 & 5104 & 2109 & 637.4 & 4.76 \\
40 & 1126 & $\mathbf{4 7 4 6}$ & 1673 & 455.7 & 3.73 \\
60 & 876 & 5606 & $\mathbf{1 6 0 5}$ & 412.0 & 2.87 \\
80 & 756 & 7831 & 1635 & 401.2 & $\mathbf{2 . 2 9}$ \\
100 & 686 & 12119 & 1710 & $\mathbf{4 0 3 . 0}$ & 2.77 \\
120 & $\mathbf{6 4 0}$ & 20045 & 1811 & 411.1 & 5.51 \\
\hline
\end{tabular}

Again we can see that both high and low speed limits on the bridge correspond to high overall pollution levels and that optimal speed limits differ for different pollutants. 
Remark: The last two simulations correspond to a realistic road engineering problem, where the optimal speed limits for a subset of roads must be established to minimise emissions of interest.

As a further example, we now demonstrate the scalability of our approach through a significantly larger network. We generated the road network in Figure 6 using SUMO random network generation facilities.

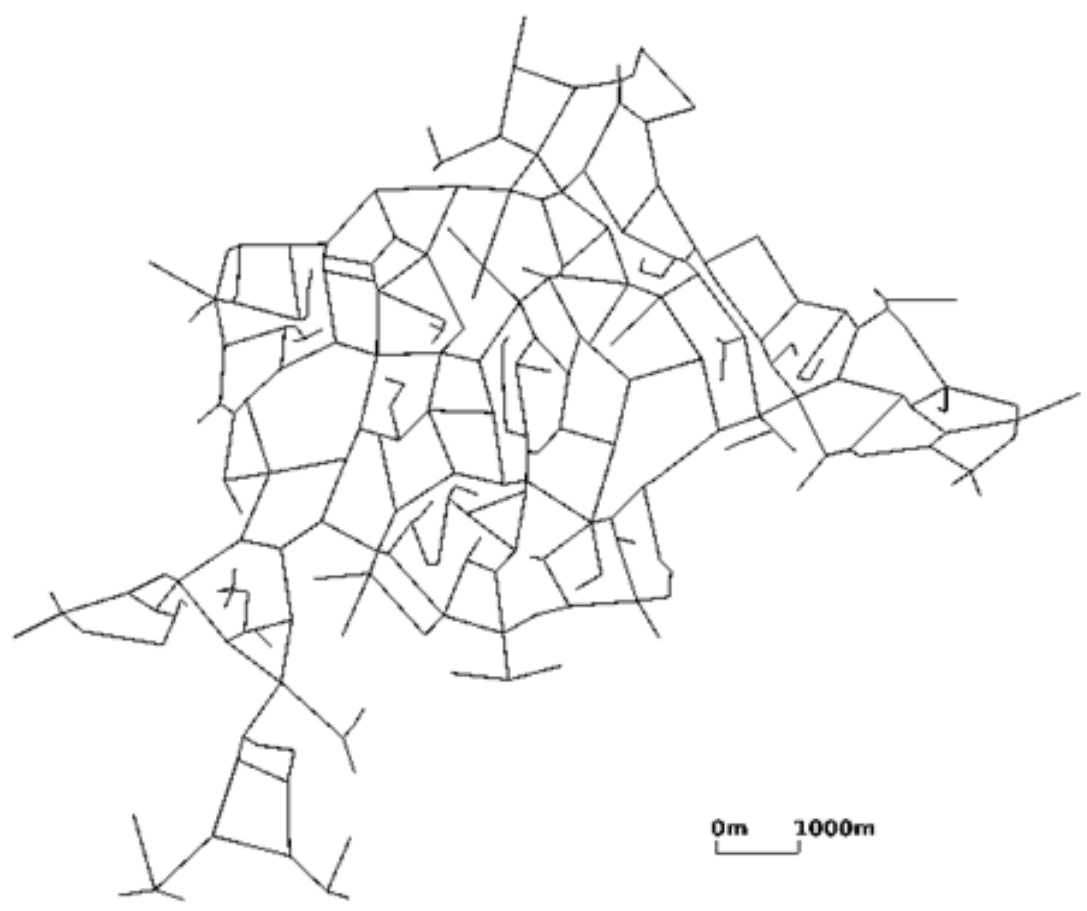

Fig. 6 A more complicated realistic road network.

Figure 7 shows the density of pollutants in the road network, together with the distribution of cars resulting from the SUMO simulation. While the road network of Figure 6 is composed of 618 road segments, only the subset of roads with ID between 250 and 300 was randomly chosen to be displayed in Figure 7, to improve readability of the result; qualitatively the distribution is the same for any selection of streets. In Figure 7 we see again that the distribution of pollutants is heterogeneous, but it is related to the distribution of cars. This is in accordance with common knowledge that congestion should affect emissions. 


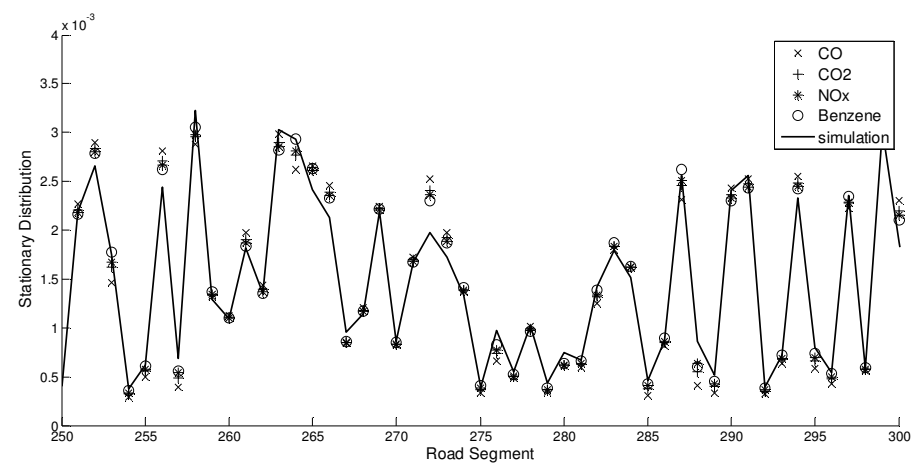

Fig. 7 Stationary distribution of time and pollutants in the more complicated example with heavy congestion. Only a selection of streets is shown to improve readability of the figure.

\section{Suggested further applications}

The primary focus of this work has been modelling. However, our principal objective remains the control of large scale urban networks. From the perspective of the road engineer, information in the Markov chain can be used for this purpose. For example, identification and avoidance of pollution peaks is a major concern in our cities. The existence of peaks can in "some sense" be identified from the Perron eigenvector of the chain, and one may reengineer the network through adjusting speed limits, traffic light sequencing, etc., so as to keep peaks away from certain sensitive spots (hospitals etc.). One may also use this information to route vehicles along low (expected) emissions paths.

Other areas to be investigated include balancing of emissions, identification of critical roads (if they close they have a bad effect on the emissions profile of the city), and the effect of fleet mixing (electric vehicles) and priority zones on the emissions profile of the urban area. Interesting observations from our model are that different pollutants require different control strategies, that low speeds do not necessarily imply low emissions, and that high speeds do not always correspond to high emissions. Future work will investigate how the conflicting objectives of low emissions for all pollutants and low congestion can be addressed in a unified framework so as to facilitate control and optimisation strategies. 


\section{Conclusions}

In this chapter we have proposed a new method of modelling urban pollutants arising from transportation networks. The efficacy of the proposed approach is demonstrated by means of a number of examples. Future work will investigate control and optimization over Markov chains (with application to road network engineering), experimental evaluation of the proposed methods, and extension of the ideas to modelling city energy profiles (with respect to routing of electric and hybrid vehicles).

Acknowledgments: SK is supported in part by the Science Foundation Ireland under Grant No. SFI/07/SK/I1216b. RS, AS and EC are supported in part by Science Foundation Ireland under grant number PI Award 07/IN.1/1901.

\section{References}

Barth M, An F, Younglove T, Scora G, Levine C, Ross M, Wenzel T (2000) Development of a comprehensive modal emissions model. NCHRP Project 25-11. Final report.

Barlow TJ, Hickman AJ, Boulter PG (2001) Exhaust emission factors 2000: Database and emission factors. Project report PR/SE/230/00. TRL Limited, Crowthorne.

Biem A, Bouillet E, Feng H, Ranganathan A, Riabov A, Verscheure O, Koutsopoulos H, Rahmani M, Güç B (2010) Real-Time Traffic Information Management using Stream Computing. IEEE Data Engineering Bulletin, 33,2.

Boulter PG, Barlow TJ, McCrae IS (2009) Emission factors 2009: Report 3 - exhaust emission factors for road vehicles in the United Kingdom. Published project report PPR356. TRL Limited.

Cho GE, Meyer CD (2001) Comparison of perturbation bounds for the stationary distribution of a Markov chain. Linear Algebra and its Applications 335:137-150.

Crisostomi E, Kirkland S, Shorten R (2011) A Google-like model of road network dynamics and its application to regulation and control. International Journal of Control, accepted for publication, 2011.

Dellnitz M, Junge O (1999) On the approximation of complicated dynamical behavior. SIAM J. Numer. Anal., 36(2):491-515.

Dellnitz M, Junge O, Koon WS, Lekien F, Lo MW, Marsden JE (2005) Transport in dynamical astronomy and multibody problems. International Journal of Bifurcation and Chaos in Applied Sciences and Engineering , no 3, 699-727.

FoE (Friends of the Earth) 1999 Road Transport, Air Pollution and Health. Briefing Paper.

Froyland G (2001) Extracting dynamical behavior via Markov models. In Nonlinear dynamics and statistics (Cambridge, 1998), Birkhäuser Boston, Boston, MA, pages 281321.

Gkatzoflias D, Kouridis, Ntziachristos L, Samaras Z (2007) COPERT IV. Computer program to calculate emissions from road transport. User Manual (version 5.0). Published by ETC-ACC (European Topic Centre on Air and Climate Change). 
Hartenstein H, Laberteaux K (2010) VANET - Vehicular applications and internetworking technologies. Wiley.

Highways Agency, Scottish Executive Development Department, Welsh Assembly Government, Department for Regional Development Northern Ireland (207). Design manual for Roads and Bridges, Volume 11, Section 3, Part 1, Air Quality, Highways Agency, Scottish Industry Department, The Welsh office and the Department of Environment for Northern Ireland, Stationary Office, London.

Huisinga W (2001) Metastability of Markovian Systems: A Transfer Operator Based Approach in Application to Molecular Dynamics. PhD thesis, Freie Universität Berlin.

Jaaskelainen J, Boethius (2009) Methodologies for assessing the impact of ITS applications on $\mathrm{CO}_{2}$ emissions. Technical Report v1.0.

Koon WS, Lo MW, Marsden JE, Ross SD (2000) Heteroclinic connections between periodic orbits and resonance transitions in celestial mechanics. Chaos, 10(2):427-469.

Koon WS, Marsden JE, Ross SD, Lo MW (2002) Constructing a low energy transfer between Jovian moons. In Celestial mechanics (Evanston, IL, 1999), volume 292 of Contemp. Math., pages 129-145. Amer. Math. Soc., Providence, RI.

Krajzewicz D, Bonert M, Wagner P (2006) The open source traffic simulation package SUMO. RoboCup 2006 Infrastructure Simulation Competition, RoboCup 2006, Bremen, Germany.

Langville AN, Meyer CD (2006) Google's PageRank and beyond - The science of search engine rankings. Princeton University Press.

Levy JI, Buonocore JJ, von Stackelberg K (2010) Evaluation of the public health impacts of traffic congestion: a health risk assessment. Environmental Health 9:65.

Meyer CD (1975) The role of group generalized inverse in the theory of finite Markov Chains. SIAM Review, 17:443-464.

Meyn SP, Tweedie RL (2009) Markov Chains and Stochastic Stability. Cambridge University Press.

Nawrot TS, Perez L, Künzli N, Munters E, Nemery B (2011) Public health importance of triggers of myocardial infarction: a comparative risk assessment. Lancet, 377:732-740.

Porta S, Crucitti P, Latora V (2006) The network analysis of urban streets: a dual approach, Physica A, 369:853-866.

Schütte C, Huisinga W (2003) Biomolecular conformations can be identified as metastable sets of molecular dynamics. In Handbook of numerical analysis, Vol. X, Handb. Numer. Anal., X. North-Holland, Amsterdam., pages 699-744 